\title{
MicroRNA regulation of BMP signaling; cross-talk between endothelium and vascular smooth muscle cells
}

Charlene Watterston, Lei Zeng, Abidemi Onabadejo, Sarah J Childs†

Alberta Children's Hospital Research Institute, and Department of Biochemistry and Molecular Biology, Cumming School of Medicine, University of Calgary, Calgary AB, Canada, T2N 4N1.

+ Corresponding author

Telephone: (403) 220-8277

Email: schilds@ucalgary.ca

Department of Biochemistry and Molecular Biology, University of Calgary, 3330 Hospital Drive NW, Calgary, AB, Canada, T2N 4N1

Statement of funding: Funding for this project derived from an NSERC grant to SJC. CW was supported by an Eyes High Studentship from the University of Calgary. LZ was supported by an Alberta Children's Hospital Research Institute Postdoctoral Fellowship and a Kertland fellowship from the Libin Cardiovascular Institute. SJC was a Canada Research Chair and an AIHS Senior Scholar.

Conflict of interest: None 


\section{Abstract}

Vascular smooth muscle cells (VSMC) are essential to the integrity of blood vessels, and therefore an attractive target of therapeutics aimed at improving vascular function. Smooth muscle cells are one of the few cell types that maintain plasticity and can switch phenotypes from differentiated (contractile) to de-differentiated (synthetic) and vice versa. As small regulatory transcripts, miRNAs act as genetic 'fine tuners' of posttranscriptional events and can act as genetic switches promoting phenotypic switching. The microRNA miR26a targets the BMP signalling effector, smad1. We show that loss of miR26 leads to hemorrhage (a loss of vascular stability) in vivo, suggesting altered vascular differentiation. Reduction in miR26a levels increases smad1 mRNA and phospho-Smad1 (pSmad1) levels. We show that increasing BMP signalling by overexpression of smad1 also leads to hemorrhage and that normalization of Smad1 levels through double knockdown of miR26 and smad1 rescues hemorrhage suggesting a direct relationship between miR26 and smad1 and vascular stability. Using a BMP genetic reporter and pSmad1 staining we show that the effect of miR26 on vascular instability is non-autonomous; BMP signalling is active in embryonic endothelial cells, but not in smooth muscle cells. Nonetheless, increased BMP signalling due to loss of miR26 results in an increase in acta2-expressing smooth muscle cell numbers and promotes a differentiated smooth muscle morphology. Taken together our data suggests that miR26 modulates BMP signalling in endothelial cells and indirectly promotes a differentiated smooth muscle phenotype. Our data also suggests that crosstalk from BMP-responsive endothelium to smooth muscle is important for its differentiation. 


\section{Introduction}

Vascular smooth muscle cells (VSMCs) provide structural integrity to the vessel wall. Guided control of signalling cascades, including Platelet Derived Growth Factor (PDGF), Notch, and Transforming Growth Factor-6/Bone morphogenic Protein (TGF-6/BMP), recruit and induce a perivascular niche of cells termed mural cells (comprising of pericytes and immature vSMCs) to create a two-layered vessel wall with an internal endothelial cell lining and a muscle cell covering (Gaengel et al. 2009; Lamont et al. 2010; Liu et al. 2007). Once the vSMCs surround the vessel they begin depositing extracellular matrix proteins such as laminin, collagen IV and fibulins to support cellular contacts (Rensen et al. 2007). vSMCs then take on a mature contractile phenotype that stabilizes the underlying endothelial cells through induction of quiescence, expression of junctional and attachment protein, and expression of contractile proteins to provide myogenic tone (Lamont et al. 2010; David et al. 2008; Owens et al. 1996; Whitesell et al. 2014).

vSMCs maintain phenotypic plasticity and can undergo a phenotypic switches from a quiescent contractile state to a proliferative synthetic state in response to various cellular stimuli (Rensen et al. 2007; Hendrix et al. 2005). Contractile vSMC are defined by an elongated, spindle-shaped morphology and low rates of proliferation. The expression of key differentiation markers such as smooth muscle $(\alpha)$ actin (Acta2), smooth muscle $\beta$-myosin heavy chain (Myh11), and transgelin (Sm22 $\alpha$ ) allows vSMCs to perform their contractile function and provide vascular tone. In contrast, rhomboid, immature (synthetic) vSMCs are highly proliferative, produce ECM proteins and have reduced expression of contractile genes (Owens et al. 2004; Mack \& Owens 1999; Miano et al. 1994; Lehoux et al. 2006).

Cumulative studies have demonstrated that BMPs and Smad1 signaling have roles in modulating VSMC plasticity (reviewed by Cai et al. 2012). Aberrant vSMCs phenotype switching plays a critical role in the pathogenesis of vascular diseases, such as hereditary hemorrhagic telangiectasia (HHT) and pulmonary arterial hypertension (PAH), with defective BMP signalling. The diseases affect both endothelial function and vSMC differentiation (Li et al. 1996; El-Bizri et al. 2008; Torihashi et al. 2009; Benjamin et al. 1998; Lan et al. 2007; Roman et al. 2002; Beppu et al. 2000; Mishina et al. 1995). Murine homozygous null mutants for BMPR-1a (Activin like kinas 3, ALK3) or the type II receptor BMPR-2 (which is mutated in human patients with PAH) (Paul et al. 2005; Orvis et al. 2008) and their ligand Bmp4 or downstream coSMAD4 are embryonic lethal, and present with vascular deformities attributable to a loss of Smad1 mediated signalling (Sirard et al. 1998). Mutations in ALK1 led to HHT2, a disease characterized by 
arteriovenous malformations (AVMs) (McDonald et al. 2011). Deletion of Alk-1 in mice leads to cranial hemorrhages, AVM-like fusion of micro-vessel plexi, dilation of large vessels and reduced coverage of vessels by vSMCs (Park et al. 2006). In fish, while disruption of Alk1 signalling results in pathological arterial enlargement and maladaptive responses generate AVMs, additional vSMCs defects have not been assessed (Corti et al. 2011).

As small noncoding RNAs, microRNAs (miRs) are genetic cues that regulate gene expression of key vSMC marker genes to control vSMC dynamics. (Albinsson et al. 2010; Ji et al. 2007; Xie et al. 2011; Zhang 2010). A number of miRNAs have been identified as modulators of the vSMC phenotype in vitro and in vivo, including miR-145, miR-21, miR-221, miR-222 and miR-146a (Zeng et al. 2009; Rangrez et al. 2011; Xu et al. 2011; Sarkar et al. 2010; Ali et al. 2015; Sun et al. 2011; Wang et al. 2010; Xin et al. 2009; Cordes et al. 2009). (Xin et al. 2009; Cordes et al. 2009; Zeng et al. 2009; Wang et al. 2010; Sarkar et al. 2010; Rangrez et al. 2011; Bai et al. 2011; Sun et al. 2011; Xu et al. 2011; Ali et al. 2015)We previously showed that miR145 promotes visceral smooth muscle differentiation via controlling cross-talk between visceral epithelial cells and smooth muscle (Zeng et al. 2009; Zeng \& Childs 2012).

In this study we investigated the role of microRNA26a (miR26a) in regulating vSMC dynamics the zebrafish model of vessel stabilization. miR26 has been identified as a potential regulator of vSMC proliferation, migration and differentiation of vSMCs in vitro (Icli et al. 2014; Leeper et al. 2011; Bai et al. 2011; Yang et al. 2017). miR-26a expression is altered during abdominal aortic aneurysm (AAA) and neointimal lesion formation (Leeper et al. 2011; Yang et al. 2017), and although primarily categorized as endothelial defects, the pathological progression and compromised vessel structure is attributed to a shift in VSMC phenotypes. However, the significance and cell autonomy of these interactions in an intact animal and in context of developing VSMC are largely unknown. Using a combination of transient knockdown methods to understand the effect of decreasing miR26a levels in vivo, we show that miR26a acts within a BMP responsive pathway to fine tune vascular smooth muscle cell maturation via targeting smad1. However, active BMP signalling was observed within endothelium in vivo, and not in smooth muscle cells. Together the evidence suggests that miR26a has a role in regulating blood vessel stabilization via a non-autonomous mechanism. 


\section{Materials and Methods:}

\section{Zebrafish}

Zebrafish (Danio Rerio) embryos were collected and incubated at $28.5{ }^{\circ} \mathrm{C}$ in E3 embryo medium and staged in hours post-fertilization (hpf) or days post fertilization (dpf). Endogenous pigmentation was inhibited from $24 \mathrm{hpf}$ by the addition of 0.003\% 1-phenyl-2-thiourea (PTU, Sigma-Aldrich) in E3 embryo medium. All animal procedures were approved by the University of Calgary Animal Care Committee. The fluorescent transgenic endothelial mCherry-expressing $T g(k d r l: m c h e r r y)^{c i 5}$, GFP-expressing $T g(k d r l: E G F P)^{l a 116}$ and $T g(f l i: n E G F P)^{y 7}\left(\right.$ Roman et al. 2002)report endothelial expression, $T g(a c t a 2: G F P)^{c a 7}$ and Tg(acta2:mCherry) ${ }^{c a 8}$ report smooth muscle expression (Whitesell et al. 2014) and BMP-reporter fish Tg(BRE-AAVmIp:eGFP) ${ }^{m w 29}$ [hereafter BRE:eGFP] report active BMP signaling (Collery \& Link 2011) .

\section{Morpholino knockdown, CRISPRi and mRNA overexpression}

Both $\mathrm{MO}$ and mimic were injected into one- to four-cell stage embryos within recommended dosage guidelines (Bill et al. 2009; Schulte-Merker \& Stainier 2014). Injected doses were 1ng/embryo for miR26 MO, Scr. control miR26, and smad1 MO. Morpholinos (MO) were obtained from Gene Tools LLC Corvalis, OR, USA. mir-26a MO blocks the mature microRNA (5-AGCCTATCCTGGATTACTTGAAC-3'), miR26a Scrambled control has 6bp mismatch (5'-ACCGTATCGTGCATTACTTCAAC-3'), and smad1 MO blocks Smad1 translation (5'-AGGAAAAGAGTGAGGTGACATTCAT-3'). For rescue experiments embryos were first injected with miR26MO and then smad1 MO. To control for non-specific neural cell death that occurs from nonspecific activation of p53 with morpholinos, a standard p53 MO was co-injected with high dose morpholino to establish dosage curve. Hsa miR26a miRIDIAN mimic was obtained from Dharmacon and injected in a dose of 3ng/embryo.

For CRISPRi mediated knockdown of miR26a, sgRNA target were designed using CHOPCHOP (Montague et al., 2014) to target the seed sequence of miR26a family members to reduce miR26 processing (miR26a-1, 26a-2, 26a-3, are independent genes located on different chromosomes. miR26b differs by one nucleotide). To generate sgRNA we followed a method established by (Narayanan et al. 2016). 10 $\mu \mathrm{mol}$ of forward primer and $50 \mu \mathrm{mol}$ of a universal reverse primer (IDT Oligos, Yale) were annealed and filled in (Montague et al. 2014), purified (Qiagen PCR purification kit) and in vitro transcribed (T7 mMessage machine, Ambion). Primer sequence information is in supplemental table 1. Zebrafish codon optimized dCas9 plasmid (Rossi et al. 2015) was linearized with Xbal and in vitro transcribed using Ambion 
Maxi Kit (Life Technologies Inc., Burlington, ON), and RNA purified using an RNeasy Mini Kit (Qiagen). Zebrafish embryos at the one-cell stage were injected with 200pg of a solution containing $75 \mathrm{ng} / \mu \mathrm{l} \mathrm{of}$ sgRNA with $150 \mathrm{ng} / \mu \mathrm{l}$ of Cas9 mRNA.

For overexpression of smad1, mRNA was in vitro transcribed as described (McReynolds et al. 2007; gift from Todd Evans Lab) using mMessage mMachine (Life Technologies Inc., Burlington, ON). 40pg of mRNA was injected per embryo at the 1 cell stage.

\section{Small molecule inhibition}

K02288 was used at a dose of 15 $\mu \mathrm{M}$ (SML1307, Sigma). DSMO (D8418, Sigma) was used as a vehicle and control. Drug stocks were heated for 20min at 65C and then diluted in E3 embryo medium. Drug or control was applied to the from $52 \mathrm{hpf}$ until $4 \mathrm{dpf}$. Embryos were grown at $28.5 \mathrm{C}$ in the dark until imaging, and drug changed once.

\section{qPCR analysis}

Total RNA from 5-10 zebrafish embryos (or heads) per treatment group was isolated using the RNeasy Mini kit for mRNA or the miRNAs Mini kit for miRNA (Qiagen). For microRNA expression, 100ng of total RNA from each sample were reverse transcribed using the miRCURY LNA ${ }^{\mathrm{TM}}$ Universal RT cDNA Synthesis Kit and expression assayed using the miRCURY LNA ${ }^{\mathrm{TM}}$ Universal RT microRNA PCR System (Qiagen). The $\Delta \Delta \mathrm{Ct}$ method was used to calculate the normalized relative expression level of a target gene from triplicate measurements. Experiments were repeated independently at least three times. Primer sequences are listed in Table S1, expression levels normalized to that of miR-103a-3p or miR122 for miRNA expression.

\section{In situ hybridization and Immunostaining}

Embryos were fixed in $4 \%$ paraformaldehyde in PBS with $0.1 \%$ Tween-20 at $4{ }^{\circ} \mathrm{C}$ overnight, followed by $100 \%$ methanol at $-20{ }^{\circ} \mathrm{C}$. For wholemount in situ hybridization embryos staining Digoxigenin (DIG)labeled antisense RNA probes. Probes for smad1 (construct describe by McReynolds et al. 2007) sm22a, acta2, myh11a were synthesized from PCR fragments amplified from embryonic zebrafish cDNA using the primers listed in supplementary table 1. Probes were synthesized by using SP6 or T7 RNA polymerase (Roche). miR26a double-DIG-labeled LNA probe was obtained from Exiqon. 
Phospho-SMAD1/5/9 (pSMAD1/5/9) was detected with Rabbit anti-Phospho-Smad1 (Ser463/465)/Smad5(Ser463/465)/Smad9(Ser426/428) (1:400; Cell Signaling Technology) using an antigen retrieval protocol, GFP was detected with mouse anti-GFP antibody, JL8 (1:500, Clontech) and detected with Alexafluor 647 or 488 secondary antibodies (1:500; Invitrogen Molecular Probes).

\section{Imaging and statistical analysis:}

For imaging, embryos were immobilized in $0.004 \%$ (g/mol) Tricaine (Sigma) and mounted in $0.8 \%$ low melt agarose on glass bottom dishes (MatTek, Ashland, MA). Confocal images were collected on a Zeiss LSM 700 inverted microscope. Image stacks were processed in Zen Blue and are presented as maximal intensity projections. For cell counts images were converted to 16-bit images using ImageJ and threshold adjusted to allow counting of cells over a region of the VA from the anterior bulbous arteriosus to the most anterior PAA.

Data are expressed as mean \pm SD. Two treatment groups were compared using Student's t-test. Three or more treatment groups were compared by one-way ANOVA followed by post hoc analysis adjusted with a least significant-difference correction for multiple comparisons using GraphPad Prism version 7.00(La Jolla California USA).

\section{Results:}

\section{miR26a is expressed in vicinity of developing blood vessels}

miR26a targets smad1 and thereby directly regulates BMP signalling (model; Figure $1 \mathrm{~A}$ ). To observe the spatial gene expression pattern of miR26a in developing embryos we used in situ hybridization. At 48hpf, miR26a has a ubiquitous expression pattern (Figure 1 B-B'), however by $4 \mathrm{dpf}$ the expression pattern becomes more localized in the ventral head of the embryo, with strong expression in the pharyngeal region, bulbous arteriosus and ventral aorta (Figure 1C). We observe that miR26a is expressed in and around the blood vessel endothelium (compare to kdrl:GFP transgenic signal; Figure $1 C^{\prime}$, inset $C^{\prime \prime}$ ) and may therefore play a role modulating BMP signalling in blood vessels. 


\section{BMP signalling is highly active in aorta endothelium of wild type embryos}

We next tested where BMP signalling was active using an in vivo reporter of Smad1/5 activity. $T g$ (BRE:eGFP] transgenic fish encode eGFP driven by an upstream $\underline{B} m p$ Response Element (BRE) that contains multiple short Smad-binding sites from the id1 promoter, a major transcriptional target activated by canonical Bmp/Smad1 signaling (Collery \& Link 2011; Laux et al. 2011). We crossed $T g$ (BRE:eGFP] to $T g$ (kdrl:mcherry) or Tg(acta2:mCherry) to localize active BMP signalling in endothelial and vSMCs respectively (Figure $1 \mathrm{D}$ and G). vSMC cells first differentiate and begin to express the mature marker acta2 between 3 and 4dpf (Whitesell et al. 2014; Georgijevic et al. 2007). Surprisingly, although miR26a has been implicated in controlling smad1 regulated vSMCs dynamics, transgenic BRE:eGFP signals were restricted to the endothelium of the vessel wall and co-expressed with kdrl:mCherry (Figure $1 \mathrm{D}, \mathrm{E}-\mathrm{D}^{\prime}$ and $\mathrm{F}-\mathrm{F}^{\prime}$ ). Differentiated acta2:mCherry-positive vSMCs lie directly adjacent to BRE:eGFPexpressing cells, with no detectable expression of BRE:eGFP in vSMCs on the ventral aorta or pharyngeal arch arteries (Figure $1 \mathrm{G}-\mathrm{G}^{\prime \prime}$ ). Similarly, acta2:mCherry- positive cells are in close association to pSmad1positive endothelial cells but do not show pSmad1 staining (Supplemental Figure 1 B-B'). Together, our data suggests, that in early development, the endothelium, but not vSMCs respond to BMP signalling as visualized by two methods of detection.

\section{Knockdown of mir26a leads to upregulation of smad1 and loss of vascular stability}

The highly conserved miR-26 family constitutes miR-26a-1, miR-26a-2, and miR-26b (Bai et al. 2011) as identified by their seed sequences and accessory sequence. In zebrafish, and humans, miR-26a-1, miR26a-2 and miR26a-3 have the same mature sequence, and only differ from the mature miR-26b sequence by two nucleotides (Icli et al. 2014; Griffiths-Jones et al. 2008). To investigate the role of miR26a in vascular development in vivo, we knocked down miR26a using an antisense morpholino that targeted the mature miRNA seed sequence of all three miR26a isoforms. A $6 \mathrm{bp}$ mismatch morpholino was used as a control. 1ng doses of morpholino were used, as suggested by current guidelines (Bill et al. 2009; Bedell et al. 2011). In parallel, we designed a second genetic knockdown approach using CRISPR interference (CRISPRi) (Long et al. 2015), to target the pri-miRNA hairpin structure using the complementary sequence to the mature miRNA (Figure $2 \mathrm{~A}$ ). RT-qPCR shows a $22 \%$ reduction in miR26 following miR26a MO knockdown and 24\% reduction with miR26a CRISPRi (Figure 2 B and C and) 
confirming that both knockdown methods result in decreased miR26a expression. The knockdown is dose-responsive; higher morpholino doses lead to a 1.8-fold reduction in miR26a expression (Supplemental Figure $3 \mathrm{~A}$ and $\mathrm{B}$ ). In support of smad1 being a target of miR26a in vivo, miR26a knockdown resulted in increased smad1 mRNA expression in $2 \mathrm{dpf}$ and $4 \mathrm{dpf}$ injected embryos as compared to controls by in situ hybridization (Figure 2D). Upregulation of smad1 was more prominent in the head of miR26a morphants and CRISPRi knockdown embryos, with a localization similar to where miR26a is expressed most strongly (Figure 1B-C). At 4dpf in both morphants and CRISPRi fish, smad1 expression is upregulated in the pharyngeal region, with staining in the ventral aorta, aortic arches and bulbous arteriosus.

In a complementary approach we injected a miR26 mimic to overexpress miR26a (Supplemental Figure 2) and observed a marked increase in miR26a expression by qPCR and in situ hybridization (Supplemental Figure $2 \mathrm{~A}, \mathrm{C}$ and D). Overexpression of miR26a resulted in mildly dorsalized embryos by $48 \mathrm{hpf}$ with heart edema, dorsal axis defects and poor circulation (Supplemental Figure 2D), suggesting overexpression of miR26 disrupts pathways such as BMP that pattern early embryonic axes.

\section{Increased levels of smad1 lead to vascular stability defects.}

At $2 \mathrm{dpf}$, miR26a morphants have an average $13 \pm 2 \%$ hemorrhage (Figure $3 \mathrm{~B}$ and D) and CRISPRi embryos have an average $15 \pm 1 \%$ hemorrhage (Figure $3 \mathrm{C}$ and $\mathrm{D}$ ). The phenotype is dose-dependent as higher doses of morpholino lead to an increase in hemorrhage to 40\% (Supplemental Figure 3 C and D). As smad1 is a known target of miR26a, but upregulation of smad1 has not been previously connected to vascular stability defects, we overexpressed smad1 by injection of mRNA into single cell stage embryos. Injection of smad1 mRNA alone resulted in significantly higher hemorrhage rate of $22 \%$ in injected embryos as compared to uninjected controls (Figure 3F and I). Next, as miR26a knockdown leads to increased smad1 levels, we predicted that reduction in smad1 would rescue hemorrhage in miR26 knockdown embryos. We used a double morpholino knockdown strategy using co-injection of a validated smad1 MO with the miR26a morpholino, both at 1ng/embryo. Double knockdown reduced hemorrhage rates to below 5\% (Figure 3H, top embryo and Figure 3I). smad1 $\mathrm{MO}$ alone does not result in hemorrhage; however it did result in a range of phenotypes associated with smad1 knockdown including dorsal-ventral axis defects and hydrocephalus as previously reported (McReynolds et al. 2007). smad1 knockdown led to $80 \%$ of embryos with a mild (V1) ventralized defect and $15 \%$ with a more severe (V2) (Figure $3 G$ and 
I). Both defects were reduced in double knockdown embryos (Figure $3 \mathrm{G}$ and $\mathrm{H}$, bottom embryos). Thus reducing miR26a levels by either MO or CRISPRi knockdown in vivo leads to an increase in smad1 levels. Increasing smad1 leads to a vascular stability defects, while reducing Smad1 levels in miR26a morphants normalizes the phenotype.

\section{Loss of miR26a leads to increased phosphorylated Smad1 in endothelium}

We next tested whether the increased expression of smad1 mRNA in miR26a knockdown embryos was also indicative of enhanced Smad1 activation by phosphorylation. Wildtype immunostaining showed pSmad1/5/9 is highest within the endothelium and not vSMCs (Supplemental Figure 1 B-B'). Using $T g$ (fli:EGFP'; $k d r l: m C h e r r y)$ transgenic embryos we counted the number of pSmad1 positive endothelial nuclei in miR26 morphants and miR26 CRISPRi knockdown embryos along the ventral aorta and from the first aortic branch of the PAA to the beginning of the bulbous arteriosus (Supplemental Figure 4). We found no significant difference in the number of endothelial cells as indicated by Fli:nEGFPy positive nuclei between the injected groups (Figure 4 A-D), however there is a significant $20 \%$ increase in the proportion of pSmad1 positive:Fliy7:nEGFP nuclei double positive endothelial cells in miR26 knockdown embryos as compared to controls, with an average of $60+3.1 \%$ and $64.9+3.9 \%$ in miR26 morphants (Figure $4 \mathrm{~F}, \mathrm{~J}$ and $\mathrm{N}$ ) and CRISPRi embryos (Figure $4 \mathrm{H}, \mathrm{L}$ and $\mathrm{O}$ ), respectively and $42-47 \%$ in controls

\section{Loss of miR26a leads to increased numbers of acta2-positive vSMCs and a differentiated morphology}

As both miR26a and smad1 are implicated in controlling vSMC differentiation, we investigated if there was a functional consequence of the increased endothelial BMP signalling on vSMCs. We assayed the expression of a key set of marker genes that are highly expressed in differentiated vSMCs. Using in situ hybridization, we found that miR26 morphant embryos have increased expression of acta2 and myh11a, especially in the pharyngeal region where vSMCs first develop (Supplementary Figure 5 A-B and C-D). This is also a similar location to that of increased smad1 staining (Figure 2D). Conversely by 4dpf miR26 mimic injected embryos had reduction in acta2 and sm22 expression (Supplementary Figure 5 E-G and $\mathrm{H}-\mathrm{I})$.

Taking advantage of live reporters, we quantitated vascular smooth muscle of the ventral aorta and pharyngeal arch arteries in miR26 morphants (Figure 5A). This assay allows us to make a number of key 
observations. First, the BRE:GFP signal appears to be enhanced in miR26a morphants (Figure $5 \mathrm{~A}^{\prime}, \mathrm{B}^{\prime}$ and C). Although this observation is not significant it correlates with increased pSmad1 staining (Figure 4). Second, the number of acta2:GFP positive cells along the ventral aorta and pharyngeal arch arteries (PAA) are increased in miR26 knockdown embryos (33.8 \pm 1.6 vs $47.22 \pm 2.2$, Figure $5 A^{\prime}, B^{\prime}$ and $\left.D\right)$. Third, the increase in acta2 positive cell number, is accompanied by a qualitative change in cell morphology of miR26a knockdown embryos. In control embryos, $25 \%$ of total acta2 positive cells have a rounded, punctate morphology, and 'sit' high on the vessel wall. The remaining cells having a more mature spindle, flattened morphology. In miR26 morphants, 13\% appear rounded while the majority, 86\%, are spindle like cells. This data suggests that loss of miR26a results in increased vSMC coverage along blood vessels that VSMCs, and a shift to a more differentiated phenotype.

\section{Inhibition of the BMP pathway reverses the effects of miR26a knockdown}

Given that pSmad1 levels are increased within the endothelium of miR26a knockdown embryos and that these embryos also have an increased number of VSMC, we investigated whether blocking BMP signalling reverses the effect of loss of miR26a on vSMC number and differentiation. We used a pharmacological approach to reduce receptor phosphorylation of Smad1. K02288 is a selective and potent inhibitor of the BMP type I receptor activin like kinase 1 (Alk1) and Alk2. To select appropriate timepoints, the endothelium of the major blood vessels are patterned by 48hpf (Isogai et al. 2001), while vSMC coverage of the ventral aorta and PAA which begins from 52hpf (Whitesell et al. 2014). Therefore, Tg(acta2:eGFP;kdrl:mCherry) embryos were treated with $15 \mu \mathrm{M}$ d K02288 from $52 \mathrm{hpf}$ to $4 \mathrm{dpf}$. Treatment at this later stage also avoids gastrulation defects and allowed us to assay the effects of BMP inhibition during a time when vSMCs first associate with blood vessels.

In wildtype embryos treatment with $\mathrm{K} 02288$ led to a $62 \%$ reduction in the total average number acta2:eGFP positive cells compared to vehicle control alone (Figure $7 \mathrm{~A}^{\prime}, \mathrm{D}^{\prime}$ and G. $29 \pm 1$ to $11+\underline{3}$ ). In miR26 knockdown embryos (Figure $7 \mathrm{~B}^{\prime}-\mathrm{C}^{\prime}$ ) the effects of $\mathrm{K} 02288$ were blunted and acta2-positive cell numbers were maintained at comparable levels to wildtype controls. miR26a morphants had a minimal $17 \%$ reduction $(35 \pm 5$ to $29 \pm 5$ ) and miR26a CRISPRi embryos had a $12 \%$ reduction in numbers from $39 \pm 1$ to $34 \pm 3$ (Figure $7 E^{\prime}-F^{\prime}$ and $G$ ). This suggests that loss of miR26a, and the subsequent increase in Smad1 may compensate for reduced receptor function and provide enough signal to maintain vSMC coverage. Alongside the reduction in vSMC number, we also observed an average $50 \%$ reduction in the length of 
ventral aorta length in K02288 treated wildtype embryos at $4 \mathrm{dpf}$, from an average $159 \mu \mathrm{m}$ to $77 \mu \mathrm{m}$ (Figure $7 \mathrm{~A}, \mathrm{D}$ and $\mathrm{H}$ ). This reduction was not seen in miR26a morphants treated with K02288, which maintain ventral aorta length. miR26a CRISPRi embryos had a small, 20\% decrease in length when treated with K02288 (Figure 7 B-C and E-F). Of note there was no statistical difference in ventral aorta length between mir26a knockdown and control embryos, which supports our finding that EC cell number is not affected. Taken together, our data indicates blocking BMP signalling effectively reduced vSMC coverage and affects endothelial development in the ventral aorta. miR26a knockdown animals have minimal changes in VSMC coverage in response to BMP inhibition.

\section{Discussion}

Compromised structural vascular integrity, vessel weakening and rupture (hemorrhage) can result from aberrant BMP signalling (Dzau et al. 2002; Milewicz et al. 2010; Nebbioso et al. 2012). Hemorrhage ultimately results from weak endothelial junctions, however defects in mural cell coverage (attachment and ECM secretion) are implicated in the pathological progression of vascular diseases. We show that the underlying endothelium of the ventral aorta in zebrafish has activated pSmad1 at 4 dpf, but that pSmad1 is not detectable in mural cells. At a stage when mature vSMC are normally present, embryos with loss of miR26a have upregulation of pSmad1, increased VSMC coverage and a change in VSMC morphology, with no observable changes in the number, or morphology of the pSmad1-expressing endothelial cells. We show that inhibition of BMP signalling reduces both vSMC coverage and the length of the ventral aorta while dual miR26 knockdown and BMP receptor inhibition leads to a rescue such that animals maintain normal VSMC number, length of the ventral aorta, and VSMC coverage. We therefore suggest that miR26a modulates BMP signalling in endothelial cells to control vSMC differentiation via yet unidentified paracrine mechanism' paracrine mechanism, and that miR26a functions to fine tune endothelial signals to the vSMCs (Figure 8).

Studies in cultured VSMC have suggested that miR26a controls Smad1-mediated BMP signalling within SMCs to modulate their phenotype (Albinsson et al. 2010). However, these in vitro studies do not address whether the levels of pathway activation in vitro are relevant to tissues in vivo. Additionally, data collected from in vitro culture systems does not address the role of cell to cell communication (autonomous and non-autonomous signalling) that is critical in vivo (Gaengel et al. 2009; Owens et al. 
2004). We therefore sought to use a live model of vascular development with intact tissue and cellular contexts. Using a BMP-reporter transgenic fish, we found that during normal development, and under physiological conditions, vSMC directly contact BRE-positive and pSmad1 positive endothelial cells but have undetectable BRE or pSmad1 signal in themselves. This important finding suggests that endothelial cells are key for responding to physiological BMP signals.

Treatment with K02288, a potent ALK1/2 inhibitor, significantly reduced both acta2-positive vSMC coverage and reduced the length of the ventral aorta. miR26 knockdown embryos showed minimal reductions in both VSMC number or VA length, which suggest that the enhanced Smad1 activation in these embryos compensates for the receptor inhibition. ALK1, ALK2 and ALK3 are expressed in both endothelial and vSMCs (Benjamin et al. 1998; Lan et al. 2007; Roman et al. 2002; Beppu et al. 2000; Mishina et al. 1995), however our data suggests that although vSMC in vitro have the potential for autonomous BMP signalling, in vivo, and under physiological conditions endothelial Smad1 activation contributes to non-autonomous vSMC coverage. In zebrafish Alk1 is highly expressed in the endothelium by $36 \mathrm{hpf}$ (Laux et al. 2011). Violet beauregarde ( $\mathrm{bg}^{\mathrm{ft09e}}$ ) alk1 loss of function zebrafish mutants develop striking cranial vessel abnormalities by $2 \mathrm{dpf}$ due to increased endothelial cell (EC) proliferation (Roman et al. 2002). $v b g^{f t 09 e}$ are also unable to limit the diameter of arteries carrying increasing flow from the heart (Corti et al. 2011). Based on our data involving indirect control by endothelial signalling, we would predict there is a defect in VSMC recruitment when Smad1 signalling downstream of Alk1 is disrupted in these mutants, although this remains to be tested.

Our data suggests that the normal function of miR26 is to reduce Smad1 protein activation within the endothelium, and indirectly inhibit vSMC differentiation in early development. Endothelial and mural cells signal through several paracrine pathways to stabilize vessels including the PGFR $\beta$, angiopoietin, TGF $\beta$, and notch pathways to modulate cell-to-cell communication (Mack 2011; Winkler et al. 2011).In one of the most important pathways for mural cell recruitment, endothelial cells release PDGF-BB ligands that acts on the mural cell receptor PDGFR- $\beta$ to initially recruit vSMCs to blood vessels (Benjamin et al. 1998; Lindahl et al. 1997). There is evidence that miR26 is modulated by PDGF-BB signalling (Yang et al. 2017). Yang et al, 2017, recently showed that neointimal hyperplasia results in elevated levels of PDGFBB and is associated with upregulation of miR26 and the accumulation of VSMC at sites of injury, indicative of increased proliferation. Furthermore, treatment of primary mouse aortic vSMCs with miR26a mimic drives cells to a synthetic vSMC state (Yang et al. 2017). Currently the link between 
increased PDGF-BB and miR26 is unknown, however because PDGF-BB is primarily released by endothelial cells, it should be explored whether it is activated downstream of Smad1 activation.

While we found increased differentiation of vSMCs at the later stage $4 \mathrm{dpf}$ timepoint, at 48hpf loss of miR26 results in hemorrhage. The $2 \mathrm{dpf}$ to $4 \mathrm{dpf}$ window is a common window for vascular instability phenotypes to emerge in zebrafish (Zheng et al. 2010; Liu et al. 2007; Montero-Balaguer et al. 2009; High et al. 2008). BMP signalling is initiated in endothelium at this timepoint and perturbations can affect endothelial cell junction development (Winkler et al. 2011). We have previously shown mural cells present around vessels by $2 \mathrm{dpf}$, although they are mesenchymal and immature(Liu et al. 2007). These cells express PDGFR $\beta$ but have no expression of mature VSMC markers (Ando et al. 2016), suggesting the 48hpf timepoint represents a critical window for vascular mural cell attachment to endothelium and differentiation to a mature phenotype. Paracrine signals from endothelium regulated downstream of miR26 could affect mural cell differentiation, leading to vascular instability.

As critical modulators of vascular cell function and with roles in cell differentiation, contraction, migration, proliferation and apoptosis, microRNAs are attractive targets of therapeutic treatments aimed at modulating the vSMC phenotypic switch. Specific to TGF- $\beta$ /BMP signalling, the mir-145/143 family has direct involvement in SMC differentiation by repressing the KIf4 to induce a contractile morphology and reduced rates of proliferation (Cordes et al., 2009). miR-21 controls vSMC differentiation through crosstalk with miR-143/-145 (Sarkar et al., 2010) and by mediating TGF- $3 / B M P$ induction to promote miR-21 cleavage to its mature form and a more contractile phenotype (Figure 5). miR26 is unique in this group in that it represses smooth muscle differentiation to mature states, likely via a paracrine signalling from endothelial cells. As drug delivery to the endothelium is relatively straightforward, modulation of miR26a might be therapeutically useful for post-transcriptional control of key genes involved in vSMC phenotypic switching. 


\section{$\underline{\text { Figures }}$}
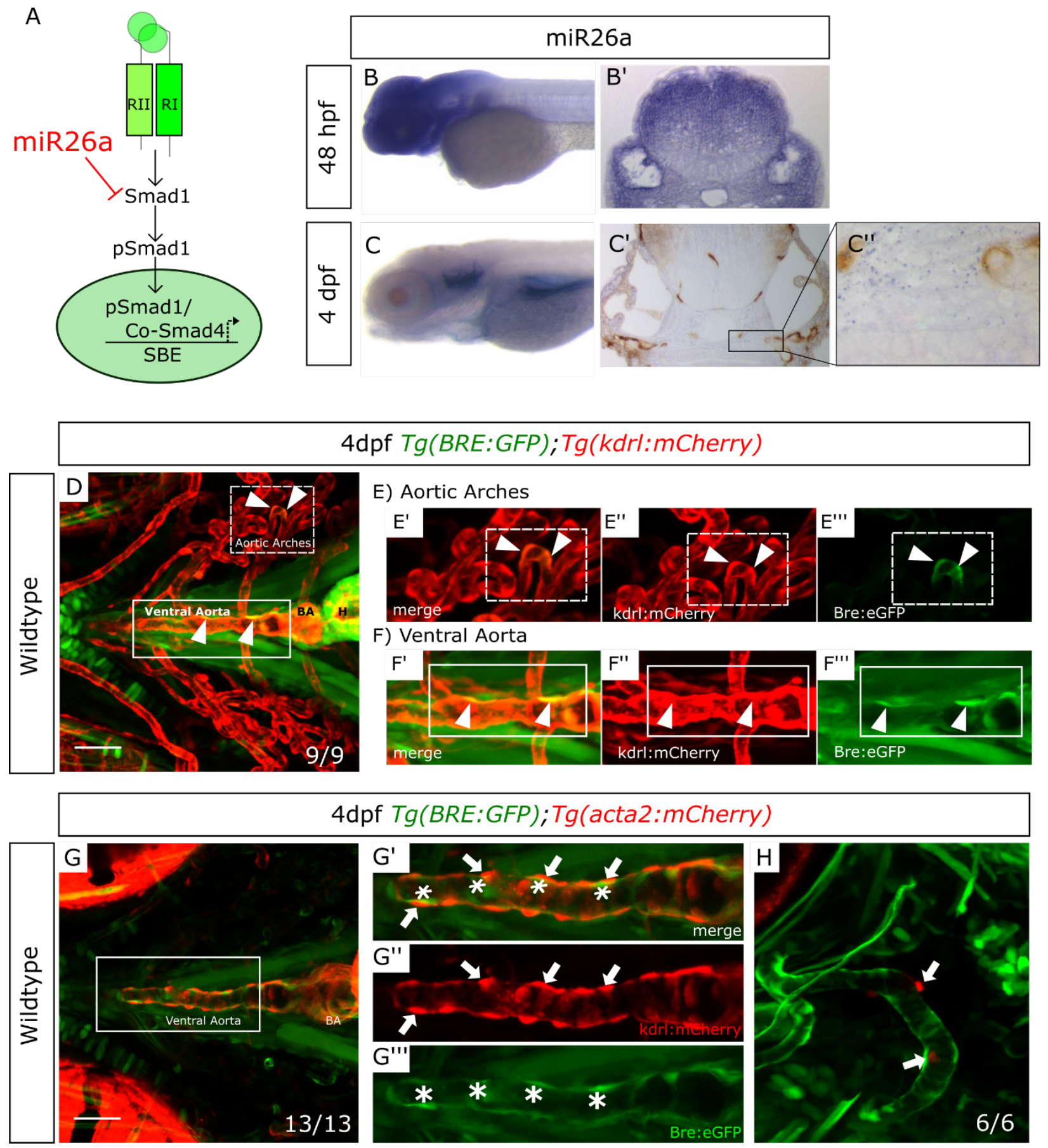

Figure 1: miR26 is expressed in the vicinity blood vessels; only endothelial cells have active BMP signaling. A) Model of how miR26a controls BMP signaling via direct targeting of smad1. In canonical Smad-mediated BMP signaling Smad1 is phosphorylated by the serine-threonine kinase activity of type $1 \mathrm{BMP}$ receptor, allowing it to associate and dimerize with the co-mediator Smad4 and translocate to the nucleus to control gene transcription. B) Lateral view of whole-mount in situ expression of miR26a at $2 \mathrm{dpf}$ shows ubiquitous expression pattern, with strong expression in the ventral head of the embryo. B' Cross section of the head at $48 \mathrm{hpf}$. C) At $4 \mathrm{dpf}$ there is strong expression of miR26 in the pharyngeal 
arches, bulbous arterious and ventral aorta. $C^{\prime}$ ) Cross section of the head showing miR26 expression in blood vessels (purple; punctate stain) compared with endothelial stain (brown; kdrl:GFP endothelial cell transgenic fish). $C^{\prime \prime}$ enlargement of image in $C^{\prime}$. D) Ventral view of the pharyngeal region of a $4 \mathrm{dpf}$ double transgenic $T g$ (BRE:eGFP); $T g(k d r l: m C h e r r y)$ embryo (BRE is in green; endothelial cells in red) shows BRE:eGFP expression within endothelial cells (arrowheads) on the $E^{\prime}-E^{\prime \prime \prime}$ ) aortic arches and $F^{\prime}-F^{\prime \prime \prime}$ ) ventral aorta. $\mathrm{G}$ and $\mathrm{H}$ ) Ventral and lateral views of a $4 \mathrm{dpf}$ double transgenic $T g$ (BRE:eGFP); $\operatorname{Tg}$ (acta2:mCherry) zebrafish shows that acta2 positive cells are in direct contact with BMP-responsive endothelial cells but do not express BRE:eGFP. Scale bar $=50 \mu \mathrm{m}$. 


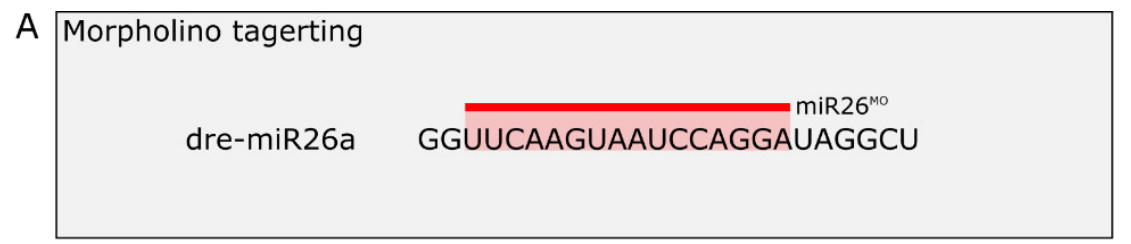

$\mathrm{B}$
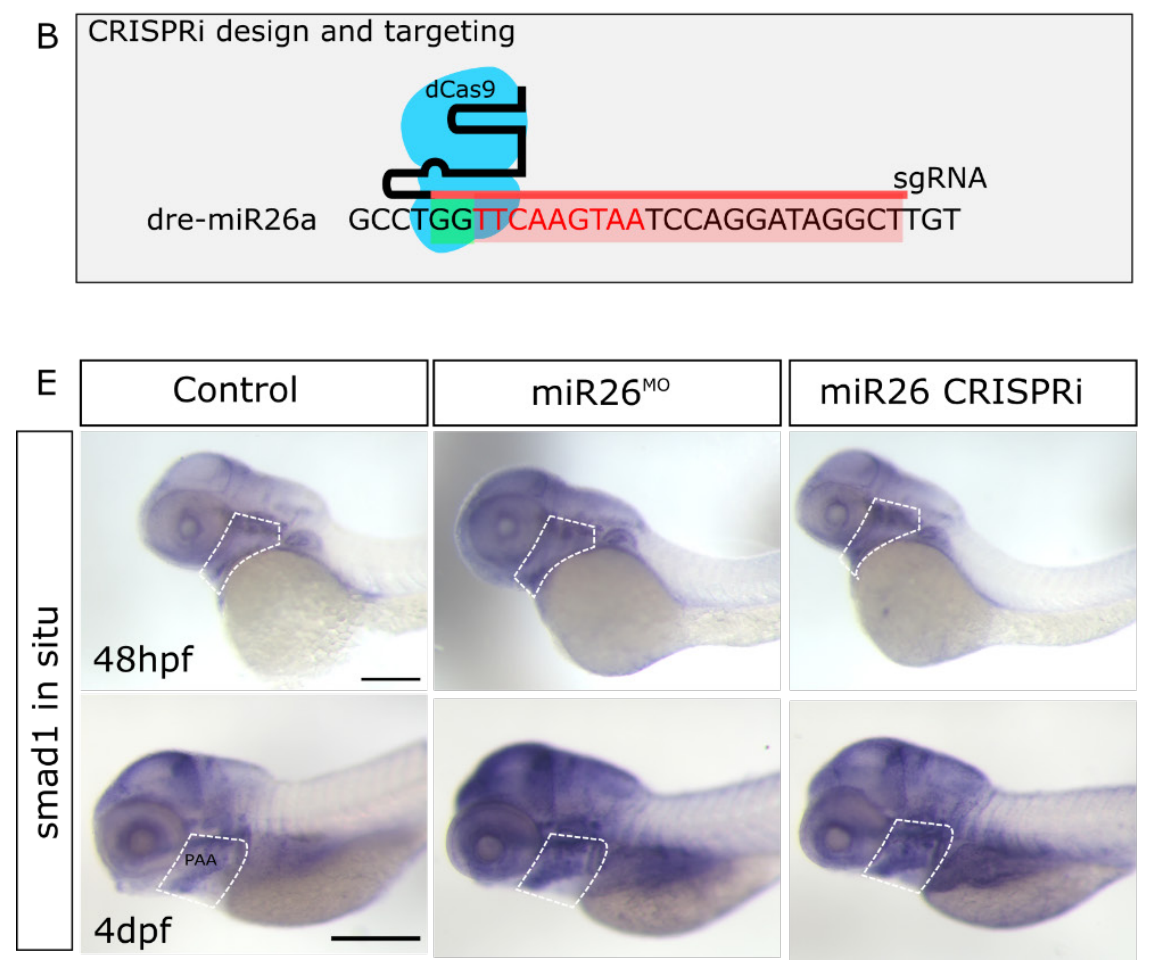
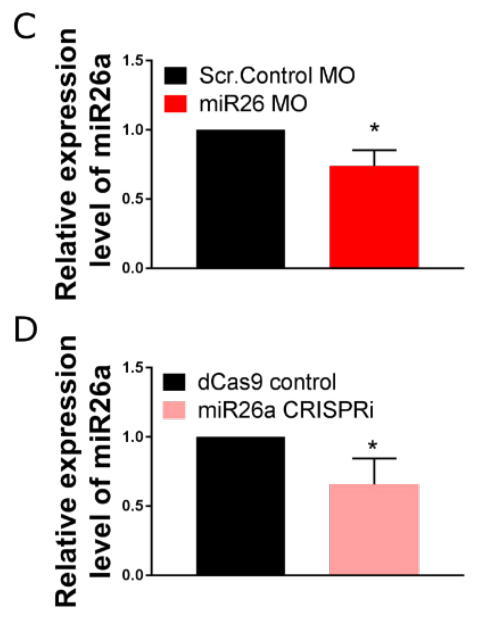

Figure 2: miR26 knockdown increases smad1 expression. A and B) Schematic of miR26 transient knockdown methods. C and D) qPCR of relative expression of miR26a in morpholino and CRISPRi injected embryos at $2 \mathrm{dpf}$, values are means of 3 replicates and normalized to miR130 expression. Error Bars = $\mathrm{SD} ; \mathrm{P}=<0.05$. E) Whole-mount in situ hybridization staining for smad1 at $2 \mathrm{dpf}$ and $4 \mathrm{dpf}$ shows increased expression of Smad1 in miR26 knockdown embryos particularly in the ventral aorta, aortic arches and pharyngeal region (dotted outline). Scale bar $=500 \mu \mathrm{m}$ 

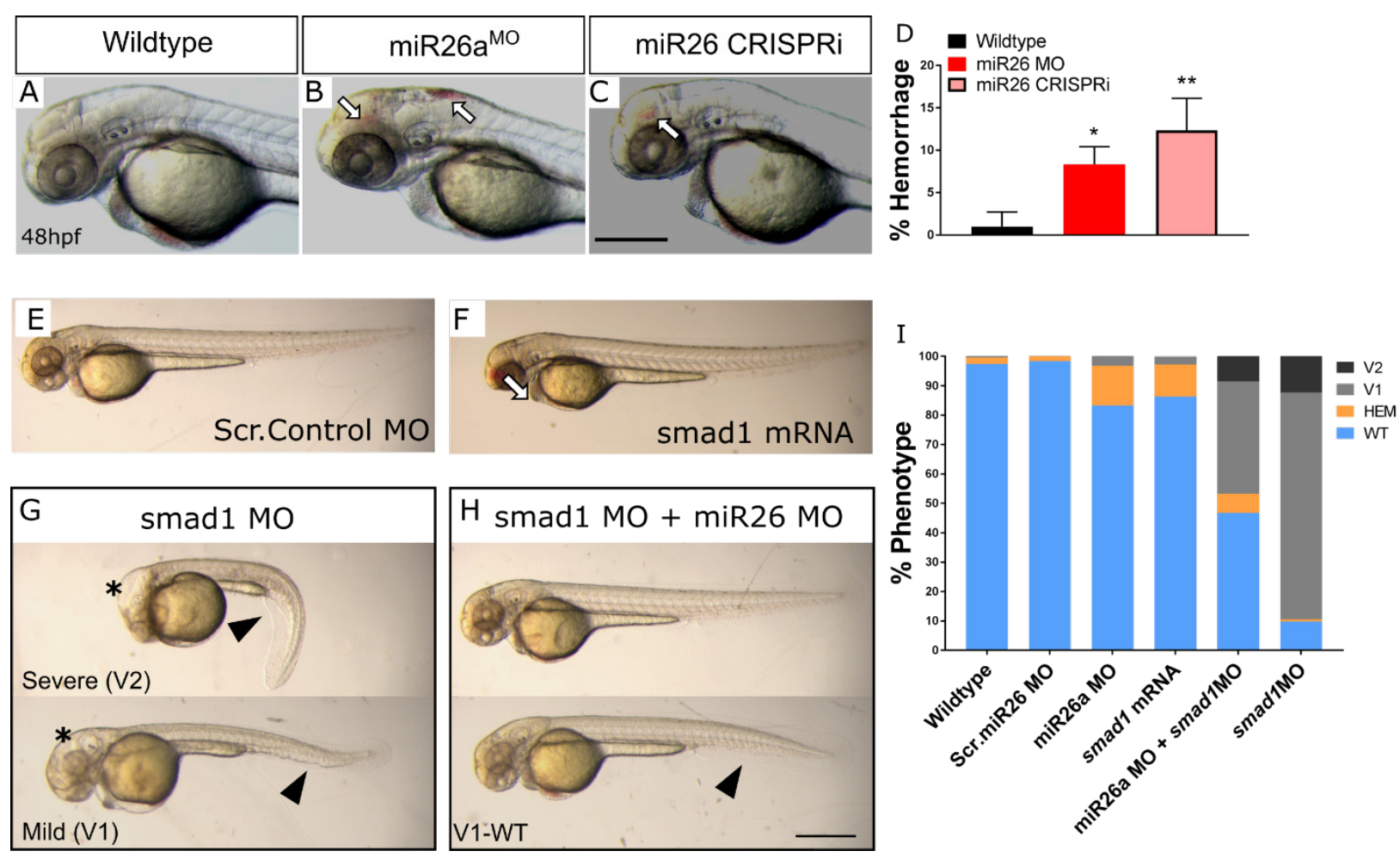

Figure 3. Increased levels of smad1 result in defects in the vascular system and body axis

A-C) Representative $2 \mathrm{dpf}$ miR26a knockdown embryos with hemorrhage, as indicated by arrows. D) Quantification of average rates of hemorrhage. (Error bars $=$ SD Unpaired t test, miR26a MO *p<0.01 and mi26 CRISPRi ${ }^{* *} p<0.001$ as compared to $W T, N=3$, Wildtype $=224$, miR26 MO $=124$, miR26 CRISPRi $=180$ ).

E-F) Representative body morphology with smad1 overexpression. G-I) miR26a and smad1 double knockdown experiments. G) Representative 2dpf smad1 MO embryos with mild (V1) and severe (V2) ventralization phenotypes. H) Representative $2 \mathrm{dpf}$ double miR26a and smad1 knockdown embryos with rescued hemorrhage and normal body axis showing only mild (V1-WT) ventralization phenotypes. I) Quantification of observed phenotypes in single and double knockdown experiments $(\mathrm{N}=4$, total $\mathrm{n}$ wildtype $=193$, Scr. Control MO $=157, \operatorname{smad} 1 \mathrm{MO}=175$, smad $1 \mathrm{mRNA}=95, \operatorname{miR} 26 \mathrm{MO}=190$, and $\operatorname{miR} 26$ $\mathrm{MO}+\operatorname{smad} 1 \mathrm{MO}=190$. Two Way ANOVA of hemorrhage phenotype. Significance: Wildtype/Scr. Control MO vs. miR26a MO $p=0.0001$ Wildtype/Scr. Control vs. SMAD1 mRNA $p=0.0001$ miR26a MO vs. miR26 MO+ SMAD1 MO p $<0.0001$ Two Way ANOVA of V1 phenotype: Wildtype/Scr. Control MO; vs. SMAD1 MO $p<0.0001$ vs. miR26 MO+ SMAD1 MO p<0.0001. Error Bars $=$ SD. Scale bar $=500 \mu \mathrm{m}$ 

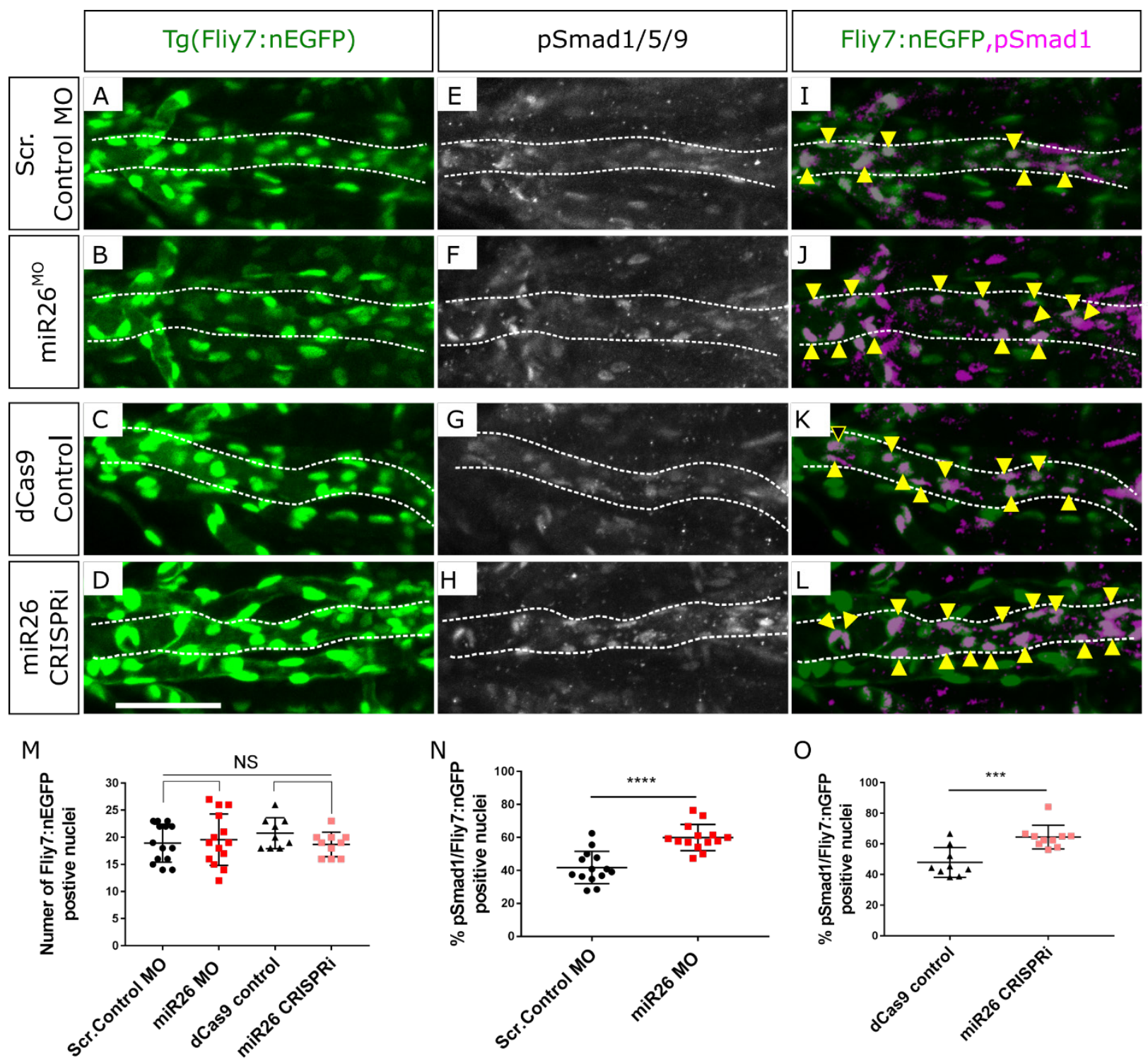

Figure 4: mir26 knockdown embryos have increased endothelial pSmad1

Ventral view confocal projections of the $4 \mathrm{dpf}$ ventral aorta (dotted outline). Endothelial nuclei (fli ${ }^{1}$ :GFP; A-D, arrowheads) and pSmad1/5/9 (white E-H) and overlay (magenta, I-L) in 4dpf Scr. Control (A,E,I), miR26a MO (B,F,J), dCas9 control $(\mathrm{C}, \mathrm{G}, \mathrm{K})$ and miR26a CRISPRi $(\mathrm{D}, \mathrm{H}, \mathrm{L})$ embryos. Yellow arrowheads indicate psmad1 + Fliy7:nEGFP double positive nuclei in the ventral aorta . M) Quantification of total number of Fliy7:nEGFP nuclei in the ventral aorta. $N$ and O) Quantification of the proportional percentage of psmad1 positive/Fliy7:nEGFP positive nuclei in miR26 morphants and miR26 CRISPRi embryos. $\mathrm{N}=3$ experiments, total embryos Scr. Control MO $=14$, miR26 MO $=14$, dCas9 control=9 and miR26a CRISRPi $=10$. Unpaired t test, $\mathrm{p}^{* * *}=0.0001$ and $\mathrm{p}^{* * * *}=0.00001$ as compared to $\mathrm{WT}$, error bars $=\mathrm{SD}$, Scale bar: $50 \mu \mathrm{m}$. 

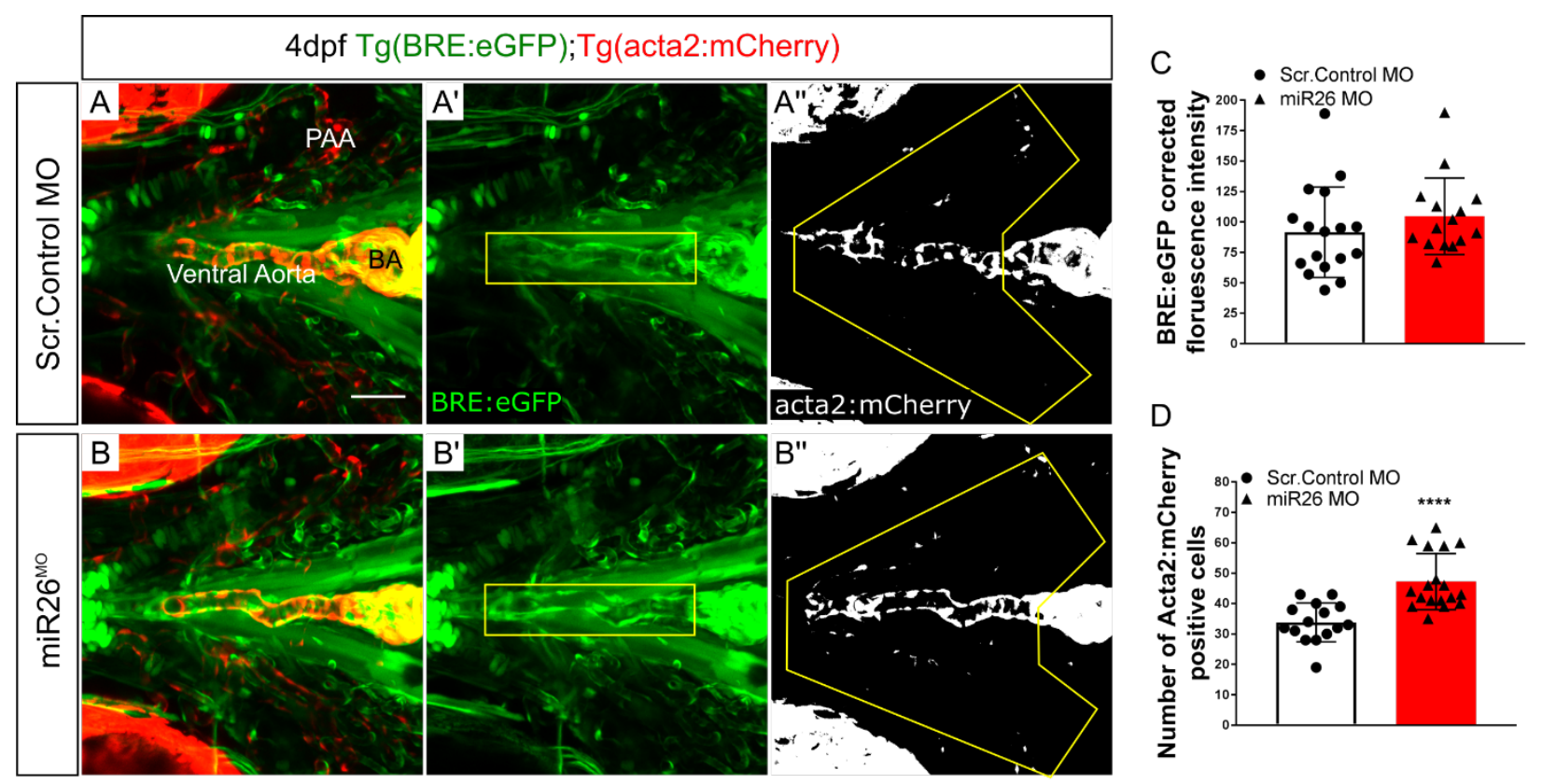

Figure 5: miR26 morphants have increased expression of vSMC genes and an increased number of acta2-positive cells

A-B) Representative orthogonal projections of ventral views of $4 \mathrm{dpf} \operatorname{Tg}(B R E$ :eGFP); $\operatorname{Tg}($ acta2:mCherry) embryos. Wildtype embryos (A-A") and miR26a morphant embryos (B-B") showing qualitative upregulation of BRE:eGFP in the ventral aorta (VA) and pharyngeal arch arteries (PAA) G) Quantification of BRE:eGFP intensity along the VA, within the yellow box in B'. H) Quantification of Acta2 positive cell number on VA and PAAs, in area outlined in $B^{\prime \prime}$. Number of acta2 positive cells is significantly increased in miR26 morphants $(\mathrm{N}=3, \operatorname{miR} 26 \mathrm{MO}=15$, Wildtype $=13$, Unpaired t test, $* * * * p<0.0001$ as compared to WT, error bars $=S D)$. 

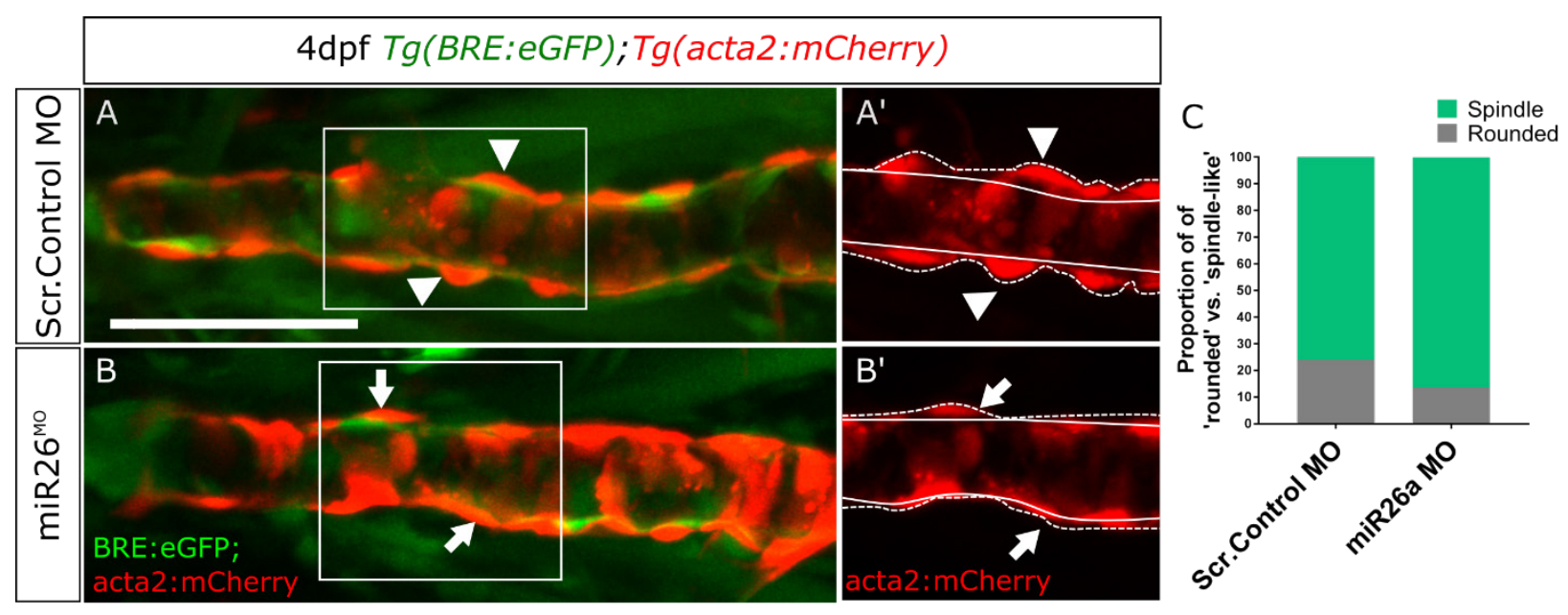

Figure 6: Trend to a more mature morphology of vascular smooth muscle cells in miR26 morphants. AB) VSMC morphology in 4dpf embryos, insets $A^{\prime}$ and $B^{\prime}$ show punctate (arrowheads) and spindle-like (arrows) morphology. Dashed lines indicate height of cells from the endothelium. C) Quantification of proportion of punctate vs spindle shaped acta 2 positive cells in wildtype and miR26 morphants. $\mathrm{N}=3$ experiments with total embryo $\mathrm{n}, \operatorname{miR} 26 \mathrm{MO}=15$, Wildtype $=13$. Scale bar $=50 \mu \mathrm{m}$ 


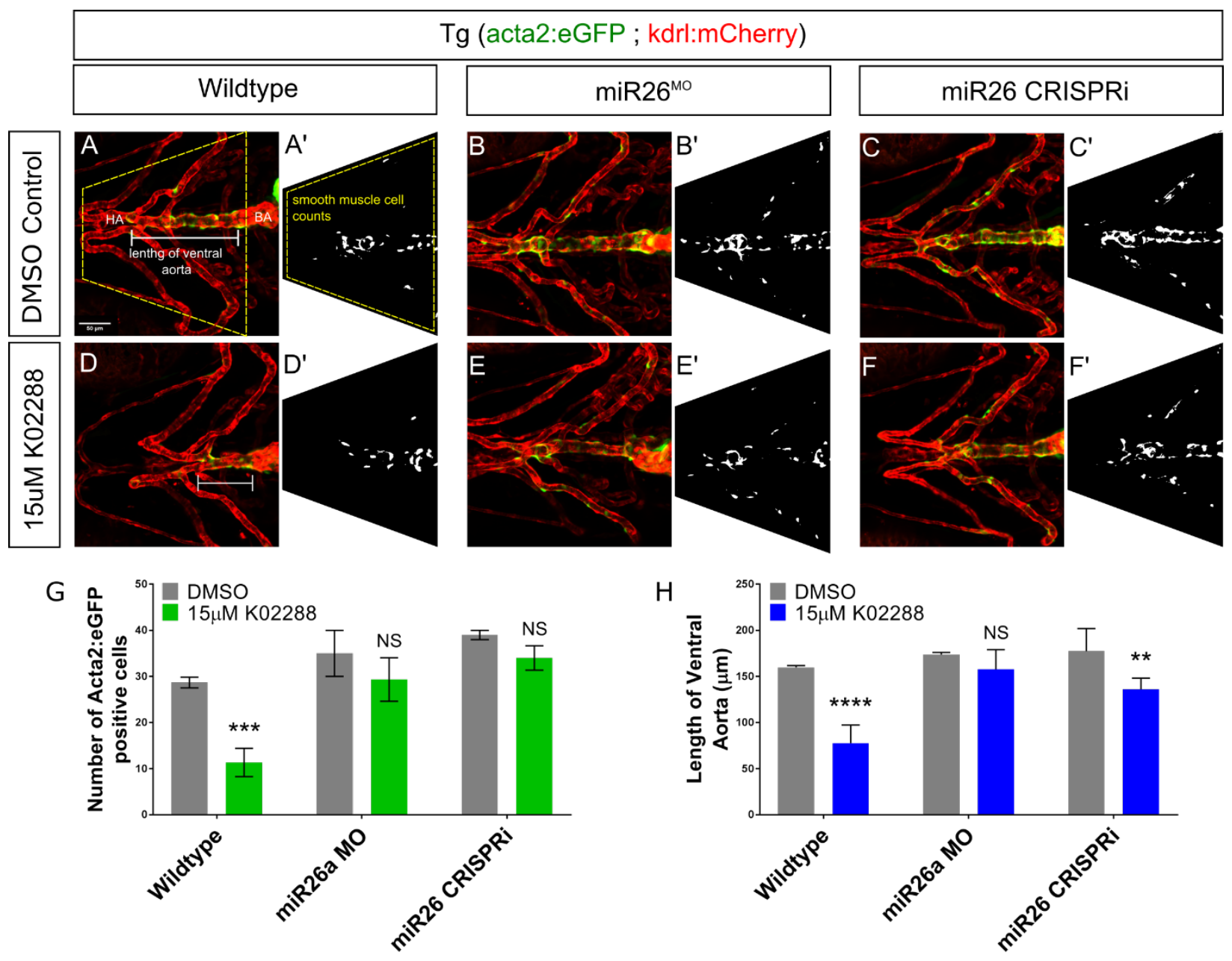

Figure 7: miR26 controls vSMC differentiation via smad1-mediated BMP signaling

Ventral aorta showing endothelial (red) and smooth muscle (green) cells in miR26 ${ }^{\mathrm{MO}}$ or CRISPRi- injected embryos treated with small molecule inhibitors from 52 hpf to $4 \mathrm{dpf}$. A-C) DMSO-treated control embryos D-F) $15 \mu \mathrm{M}$ K02288 treated control embryos. (A,D), miR26 morphant (B,E), miR26 CRISPRi knockdown (C,F). A'-F') Threshold adjusted images of acta2-eGFP expression. ( $N=3,8-9$ embryos per treatment group. One Way ANOVA, $* *, * * *, * * * * p=0.001-0.00001$. Scale bar $=50 \mu \mathrm{m}$ 

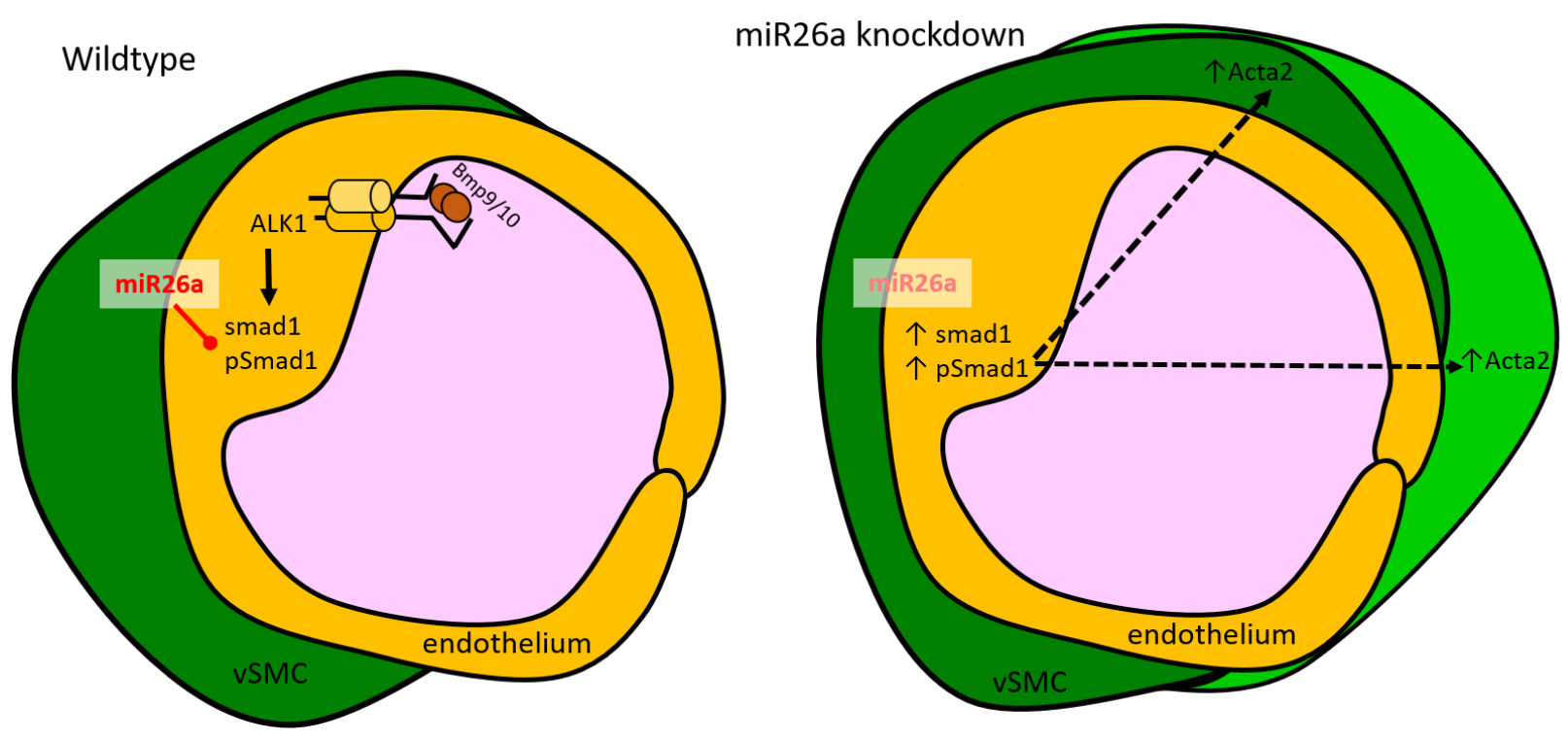

Figure 8: Proposed mechanism by which miR26a modulates BMP signaling to promote SMC phenotype via interactions with endothelial cells

miR26 modulates vascular stability by directly targeting smad1. At developmental stages when smooth muscle appears, the endothelium has active BMP signaling. Loss of miR26 results in increased BMP signaling in endothelial cells where smad1 becomes phosphorylated. Increased pSmad1 in endothelial cells leads to increased acta 2 and increased acta2-positive VSMC cell number, while blocking BMP signaling leads to a decrease of both. 


\section{$\underline{\text { References }}$}

Albinsson, S. et al., 2010. miRNAs are necessary for vascular smooth muscle growth, differentiation and function. Arterioscler Thromb Vasc Biol, 30(6), pp.1118-1126.

Ali, S.S. et al., 2015. Pathological microRNAs in acute cardiovascular diseases and microRNA therapeutics. Journal of Acute Disease. Available at: http://www.sciencedirect.com/science/article/pii/S2221618915000797 [Accessed October 26, 2015].

Ando, K. et al., 2016. Clarification of mural cell coverage of vascular endothelial cells by live imaging of zebrafish. Development (Cambridge, England), 143(8), pp.1328-1339.

Bai, Y. et al., 2011. Circulating microRNA-26a: potential predictors and therapeutic targets for nonhypertensive intracerebral hemorrhage. Med Hypotheses, 77(4), pp.488-490.

Bedell, V.M., Westcot, S.E. \& Ekker, S.C., 2011. Lessons from morpholino-based screening in zebrafish. Brief Funct Genomics, 10(4), pp.181-188.

Benjamin, L.E., Hemo, I. \& Keshet, E., 1998. A plasticity window for blood vessel remodelling is defined by pericyte coverage of the preformed endothelial network and is regulated by PDGF-B and VEGF. Development, 125(9), pp.1591-1598.

Beppu, H. et al., 2000. BMP type II receptor is required for gastrulation and early development of mouse embryos. Developmental biology, 221(1), pp.249-58. Available at:

http://www.sciencedirect.com/science/article/pii/S0012160600996702 [Accessed May 24, 2016].

Bill, B.R. et al., 2009. A primer for morpholino use in zebrafish. Zebrafish, 6(1), pp.69-77.

Cai, J. et al., 2012. BMP signaling in vascular diseases. FEBS Letters, 586(14), pp.1993-2002. Available at: https://www.sciencedirect.com/science/article/pii/S0014579312003195\#b0785 [Accessed August 1, 2018].

Collery, R.F. \& Link, B.A., 2011. Dynamic Smad-mediated BMP signaling revealed through transgenic zebrafish. Developmental dynamics : an official publication of the American Association of Anatomists, 240(3), pp.712-722.

Cordes, K.R. et al., 2009. miR-145 and miR-143 regulate smooth muscle cell fate and plasticity. Nature, 460(7256), pp.705-710.

Corti, P. et al., 2011. Interaction between alk1 and blood flow in the development of arteriovenous malformations. Development, 138(8), pp.1573-1582. Available at: http://dev.biologists.org/content/138/8/1573.abstract.

David, L. et al., 2008. Bone morphogenetic protein-9 is a circulating vascular quiescence factor. Circ Res, 102. Available at: http://dx.doi.org/10.1161/CIRCRESAHA.107.165530.

Dzau, V.J., Braun-Dullaeus, R.C. \& Sedding, D.G., 2002. Vascular proliferation and atherosclerosis: new perspectives and therapeutic strategies. Nat Med, 8(11), pp.1249-1256.

El-Bizri, N. et al., 2008. SM22 $\alpha$-Targeted Deletion of Bone Morphogenetic Protein Receptor IA in Mice 
Impairs Cardiac and Vascular Development and Influences Organogenesis. Development (Cambridge, England), 135(17), pp.2981-2991.

Gaengel, K. et al., 2009. Endothelial-mural cell signaling in vascular development and angiogenesis. Arteriosclerosis, thrombosis, and vascular biology, 29(5), pp.630-638.

Georgijevic, S. et al., 2007. Spatiotemporal expression of smooth muscle markers in developing zebrafish gut. Developmental Dynamics, 236(6), pp.1623-1632.

Griffiths-Jones, S. et al., 2008. miRBase: tools for microRNA genomics. Nucleic acids research, 36(suppl 1), pp.D154-D158.

Hendrix, J.A. et al., 2005. 5' CArG degeneracy in smooth muscle $\alpha$-actin is required for injury-induced gene suppression in vivo. Journal of Clinical Investigation, 115(2), pp.418-427.

High, F.A. et al., 2008. Endothelial expression of the Notch ligand Jagged1 is required for vascular smooth muscle development. Proceedings of the National Academy of Sciences, 105(6), p.1955 LP1959. Available at: http://www.pnas.org/content/105/6/1955.abstract.

Icli, B., Dorbala, P. \& Feinberg, M.W., 2014. An emerging role for the miR-26 family in cardiovascular disease. Trends in cardiovascular medicine, 24(6), pp.241-248.

Isogai, S., Horiguchi, M. \& Weinstein, B.M., 2001. The Vascular Anatomy of the Developing Zebrafish: An Atlas of Embryonic and Early Larval Development. Developmental Biology, 230(2), pp.278-301. Available at: http://www.sciencedirect.com/science/article/pii/S0012160600999950.

Ji, R. et al., 2007. MicroRNA expression signature and antisense-mediated depletion reveal an essential role of MicroRNA in vascular neointimal lesion formation. Circ Res, 100(11), pp.1579-1588.

Lamont, R.E. et al., 2010. Hedgehog signaling via angiopoietin1 is required for developmental vascular stability. Mechanisms of development, 127(3), pp.159-168.

Lan, Y. et al., 2007. Essential role of endothelial Smad4 in vascular remodeling and integrity. Molecular and cellular biology, 27(21), pp.7683-7692.

Laux, D.W., Febbo, J.A. \& Roman, B.L., 2011. Dynamic analysis of BMP-responsive smad activity in live zebrafish embryos. Developmental Dynamics, 240(3), pp.682-694. Available at: http://dx.doi.org/10.1002/dvdy.22558.

Leeper, N.J. et al., 2011. MicroRNA-26a is a novel regulator of vascular smooth muscle cell function. Journal of cellular physiology, 226(4), pp.1035-1043.

Lehoux, S., Castier, Y. \& Tedgui, A., 2006. Molecular mechanisms of the vascular responses to haemodynamic forces. Journal of internal medicine, 259(4), pp.381-392.

Li, L. et al., 1996. Expression of the SM22alpha promoter in transgenic mice provides evidence for distinct transcriptional regulatory programs in vascular and visceral smooth muscle cells. The Journal of cell biology, 132(5), pp.849-859.

Lindahl, P. et al., 1997. Pericyte loss and microaneurysm formation in PDGF-B-deficient mice. Science, 277(5323), pp.242-245.

Liu, J. et al., 2007. A ßPix-Pak2a signaling pathway regulates cerebral vascular stability in zebrafish. 
Proceedings of the National Academy of Sciences, 104(35), pp.13990-13995.

Long, L. et al., 2015. Regulation of transcriptionally active genes via the catalytically inactive Cas9 in C. elegans and D. rerio. Cell research, 25(5), p.638.

Mack, C.P., 2011. Signaling Mechanisms That Regulate Smooth Muscle Cell Differentiation. Arteriosclerosis, thrombosis, and vascular biology, 31(7), pp.1495-1505.

Mack, C.P. \& Owens, G.K., 1999. Regulation of smooth muscle $\alpha$-actin expression in vivo is dependent on CArG elements within the $5^{\prime}$ and first intron promoter regions. Circulation Research, 84(7), pp.852-861.

McDonald, J., Bayrak-Toydemir, P. \& Pyeritz, R.E., 2011. Hereditary hemorrhagic telangiectasia: An overview of diagnosis, management, and pathogenesis. Genetics In Medicine, 13, p.607. Available at: http://dx.doi.org/10.1097/GIM.0b013e3182136d32.

McReynolds, L.J. et al., 2007. Smad1 and Smad5 differentially regulate embryonic hematopoiesis. Blood, 110(12), pp.3881-3890.

Miano, J.M. et al., 1994. Smooth muscle myosin heavy chain exclusively marks the smooth muscle lineage during mouse embryogenesis. Circulation research, 75(5), pp.803-812.

Milewicz, D.M. et al., 2010. Genetic variants promoting smooth muscle cell proliferation can result in diffuse and diverse vascular diseases: evidence for a hyperplastic vasculomyopathy. Genetics IN Medicine, 12(4), pp.196-203.

Mishina, Y. et al., 1995. Bmpr encodes a type I bone morphogenetic protein receptor that is essential for gastrulation during mouse embryogenesis. Genes \& development, 9(24), pp.3027-3037.

Montague, T.G. et al., 2014. CHOPCHOP: a CRISPR/Cas9 and TALEN web tool for genome editing. Nucleic acids research, p.gku410.

Montero-Balaguer, M. et al., 2009. Stable Vascular Connections and Remodeling Require Full Expression of VE-Cadherin in Zebrafish Embryos. PLOS ONE, 4(6), p.e5772. Available at: https://doi.org/10.1371/journal.pone.0005772.

Narayanan, A. et al., 2016. In vivo mutagenesis of miRNA gene families using a scalable multiplexed CRISPR/Cas9 nuclease system. Scientific Reports, 6, p.32386. Available at: http://dx.doi.org/10.1038/srep32386.

Nebbioso, A. et al., 2012. Trials with "epigenetic" drugs: an update. Molecular oncology, 6(6), pp.65782. Available at: http://www.moloncol.org/article/S1574789112000968/fulltext [Accessed July 13, 2015].

Orvis, G.D. et al., 2008. Functional redundancy of tgf-Beta family type I receptors and receptor-smads in mediating anti-mullerian hormone-induced mullerian duct regression in the mouse. Biol Reprod, 78. Available at: http://dx.doi.org/10.1095/biolreprod.107.066605.

Owens, G.K., Kumar, M.S. \& Wamhoff, B.R., 2004. Molecular regulation of vascular smooth muscle cell differentiation in development and disease. Physiological reviews, 84(3), pp.767-801. Available at: http://physrev.physiology.org/content/84/3/767 [Accessed March 8, 2015]. 
Owens, G.K., Vernon, S.M. \& Madsen, C.S., 1996. Molecular regulation of smooth muscle cell differentiation. Journal of hypertension. Supplement: official journal of the International Society of Hypertension, 14(5), pp.S55-64.

Park, C. et al., 2006. Bone morphogenetic protein receptor $1 \mathrm{~A}$ signaling is dispensable for hematopoietic development but essential for vessel and atrioventricular endocardial cushion formation. Development, 133. Available at: http://dx.doi.org/10.1242/dev.02499.

Paul, B.Y. et al., 2005. Bone morphogenetic protein (BMP) type II receptor deletion reveals BMP ligandspecific gain of signaling in pulmonary artery smooth muscle cells. Journal of Biological Chemistry, 280(26), pp.24443-24450.

Rangrez, A.Y. et al., 2011. MiR-143 and miR-145 molecular keys to switch the phenotype of vascular smooth muscle cells. Circulation: Cardiovascular Genetics, 4(2), pp.197-205.

Rensen, S.S.M., Doevendans, P. \& Van Eys, G., 2007. Regulation and characteristics of vascular smooth muscle cell phenotypic diversity. Netherlands Heart Journal, 15(3), pp.100-108.

Roman, B.L. et al., 2002. Disruption of acvrl1 increases endothelial cell number in zebrafish cranial vessels. Development, 129(12), pp.3009-3019. Available at:

http://dev.biologists.org/content/129/12/3009.abstract.

Rossi, A. et al., 2015. Genetic compensation induced by deleterious mutations but not gene knockdowns. Nature, 524, p.230. Available at: http://dx.doi.org/10.1038/nature14580.

Sarkar, J. et al., 2010. MicroRNA-21 plays a role in hypoxia-mediated pulmonary artery smooth muscle cell proliferation and migration. American Journal of Physiology-Lung Cellular and Molecular Physiology, 299(6), pp.L861-L871.

Schulte-Merker, S. \& Stainier, D.Y.R., 2014. Out with the old, in with the new: reassessing morpholino knockdowns in light of genome editing technology. Development, 141(16), pp.3103-3104.

Sirard, C. et al., 1998. The tumor suppressor gene Smad4/Dpc4 is required for gastrulation and later for anterior development of the mouse embryo. Genes \& development, 12(1), pp.107-119.

Sun, S. et al., 2011. miR-146a and Krüppel-like factor 4 form a feedback loop to participate in vascular smooth muscle cell proliferation. EMBO reports, 12(1), pp.56-62.

Torihashi, S. et al., 2009. The expression and crucial roles of BMP signaling in development of smooth muscle progenitor cells in the mouse embryonic gut. Differentiation; research in biological diversity, 77(3), pp.277-89. Available at: http://www.sciencedirect.com/science/article/pii/S0301468108000261 [Accessed May 10, 2016].

Wang, J. et al., 2010. Serum miR-146a and miR-223 as potential new biomarkers for sepsis. Biochemical and biophysical research communications, 394(1), pp.184-188.

Whitesell, T.R. et al., 2014. An ?-Smooth Muscle Actin (acta2/?sma) Zebrafish Transgenic Line Marking Vascular Mural Cells and Visceral Smooth Muscle Cells. PLOS ONE, 9(3), p.e90590. Available at: http://dx.doi.org/10.1371\%2Fjournal.pone.0090590.

Winkler, E.A., Bell, R.D. \& Zlokovic, B.V., 2011. Lack of Smad or Notch Leads to a Fatal Game of Brain Pericyte Hopscotch. Developmental Cell, 20(3), pp.279-280. Available at: 
https://www.sciencedirect.com/science/article/pii/S1534580711000840 [Accessed August 1, 2018].

Xie, C., Zhang, J. \& Chen, Y.E., 2011. MicroRNA and vascular smooth muscle cells. Vitam Horm, 87, pp.321-339.

Xin, M. et al., 2009. MicroRNAs miR-143 and miR-145 modulate cytoskeletal dynamics and responsiveness of smooth muscle cells to injury. Genes \& Development . Available at: http://genesdev.cshlp.org/content/early/2009/08/31/gad.1842409.abstract.

Xu, J. et al., 2011. Circulating MicroRNAs, miR-21, miR-122, and miR-223, in patients with hepatocellular carcinoma or chronic hepatitis. Molecular carcinogenesis, 50(2), pp.136-142.

Yang, X. et al., 2017. MiR-26a contributes to the PDGF-BB-induced phenotypic switch of vascular smooth muscle cells by suppressing Smad1. Oncotarget, 8(44), pp.75844-75853.

Zeng, L., Carter, A.D. \& Childs, S.J., 2009. miR-145 directs intestinal maturation in zebrafish. Proceedings of the National Academy of Sciences , 106(42), pp.17793-17798. Available at: http://www.pnas.org/content/106/42/17793.abstract.

Zeng, L. \& Childs, S.J., 2012. The smooth muscle microRNA miR-145 regulates gut epithelial development via a paracrine mechanism. Developmental Biology, 367(2), pp.178-186.

Zhang, C., 2010. MicroRNAs in vascular biology and vascular disease. Journal of cardiovascular translational research, 3(3), pp.235-240.

Zheng, X. et al., 2010. CCM3 signaling through sterile 20-like kinases plays an essential role during zebrafish cardiovascular development and cerebral cavernous malformations. The Journal of Clinical Investigation, 120(8), pp.2795-2804. Available at: https://doi.org/10.1172/JCl39679. 